\title{
The DAMNED Simulator for Implementing a Dynamic Model of the Network Controlling Saccadic Eye Movements
}

\author{
Anthony Mouraud $^{1,3}$, Alain Guillaume ${ }^{2,3}$, and Hélène Paugam-Moisy ${ }^{1,4}$ \\ ${ }^{1}$ LIRIS, UMR CNRS 5205, Université de Lyon, F-69676 Bron, France \\ ${ }^{2}$ LNC, UMR CNRS 6155, Université de Provence, F-13331 Marseille, France \\ ${ }^{3}$ Fac. Sc. Sport, Université de la Méditerranée, F-13288 Marseille, France \\ ${ }^{4}$ INRIA-Saclay, LRI, Université Paris Sud 11, F-91405 Orsay, France, \\ anthony.mouraudegmail.com, alain.guillaumedunivmed.fr, helene. \\ paugam-moisy@univ-lyon2.fr
}

\begin{abstract}
The DAMNED simulator is a Distributed And Multithreaded Neural Event-Driven framework designed for implementing Spiking Neuron Networks (SNNs). This paper shows the power of DAMNED for simulating the temporal dynamics of a biologically inspired model of the system controlling saccadic eye movements. A fundamental neural structure for the saccade generation is the Superior Colliculus (SC). The proposed model relies on two pathways leaving this structure: A first one supervises the motor error and the movement initiation and a second one provides a direct drive to premotor centers. This simple model, its SNN implementation and its dynamic behaviour reproduce the evolution of movement amplitude as a function of activity location in the SC. It also accounts for classical results obtained when the SC is subjected to electrical stimulations.
\end{abstract}

Key words: spiking neuron network, event-driven simulation, distributed simulation, saccadic eye movement control.

\section{Introduction}

Many models are proposed by neuroscientists for explaining how the neural activity could be the basis of behavioural control or cognitive mechanisms. The models are mostly defined by a scheme on a paper, and they are very useful for designing experiments on human or animals, but they are seldom implemented in a way that may show how they work and behave through time. A gap is thus induced between models and experiments, the latter accounting more and more for temporal recordings. However, Spiking Neuron Networks (SNNs) are excellent candidates for simulating the dynamics of interactions between several neural substrates in a biologically plausible way.

The DAMNED simulator has been designed for realizing simulations of biologically plausible large scale SNNs in a distributed and event-driven way [1]. The present paper proposes to take advantage of the DAMNED simulator to implement and analyze the dynamic behaviour of a theoretical model of the brainstem neural network involved 
in the saccadic eye movements generation. Saccades are the high velocity displacements of the eye produced to align the greatest accuracy zone of the retina, the fovea, with targets of interest. The saccadic part of the oculomotor system has been studied since decades through animal and human experiments [2-4]. A fundamental characteristic of this system is its organization in a feedback loop that would control the amplitude of the saccade [2]. The precise description of the neural circuit that produces saccades has been attractive for modelling. Many models of the saccadic system have already been proposed [5] but none of them has been implemented with spiking neuron networks. All these computational models have brought both informations and hypotheses about the interactions of the underlying neural networks. In the present paper we propose a slightly different model that tests a new hypothesis for the organization of the feedback loop in the brainstem. For validation, the model has been implemented in the form of a modular SNN and its dynamics has been simulated with DAMNED.

Section 2 briefly outlines the simulator characteristics. Section 3 presents the model proposed for the sub-collicular saccade generation system. Section 4 details the experimental protocols applied to the SNN simulating the model and the functionalities of the saccadic system studied in simulation. Section 5 comments the behaviour observed through time in the eye movement control system and discusses the model validation.

\section{Simulation Framework}

\subsection{The DAMNED Simulator}

DAMNED is not the only spiking neuron networks simulator but to our best knowledge DAMNED is the only one that gathers event-driven, distributed and multithreaded characteristics as the basis of its design [6]. The principles of the DAMNED simulation framework have been defined in [1]. The computational performance of the simulator has been tested on a common hardware architecture and analyzed for large scale SNNs [7].

The simulator takes advantage of both distributed hardware and concurrent threads. Communications via message passing between CPUs (multi-core, computer network, cluster or parallel machine), and also local concurrency between calculation and communication (threads), speed up the computation and enlarge the available memory, compared to sequential SNN simulation. Figure 1 proposes a layered view of the DAMNED global architecture. The first layer is the distributed hardware (processor/memory couples). DAMNED achieves communications between hardware resources via the Message Passing Interface (MPI) with ssh (secured shell) for the message delivery (second and third layers). In case of a multiprocessor hardware, messages are passed through the local bus. For each MPI node the DAMNED simulator creates two threads (fourth layer): A CoMmunication Controller (CMC) sends and receives spike event messages and a ComPutation Controller (CPC) allocates each time-stamped spike event to an Event-driven Cell (EC), aka the target spiking neuron that computes the event and eventually creates a new spike event in response. The last layer is the SNN to be simulated. 


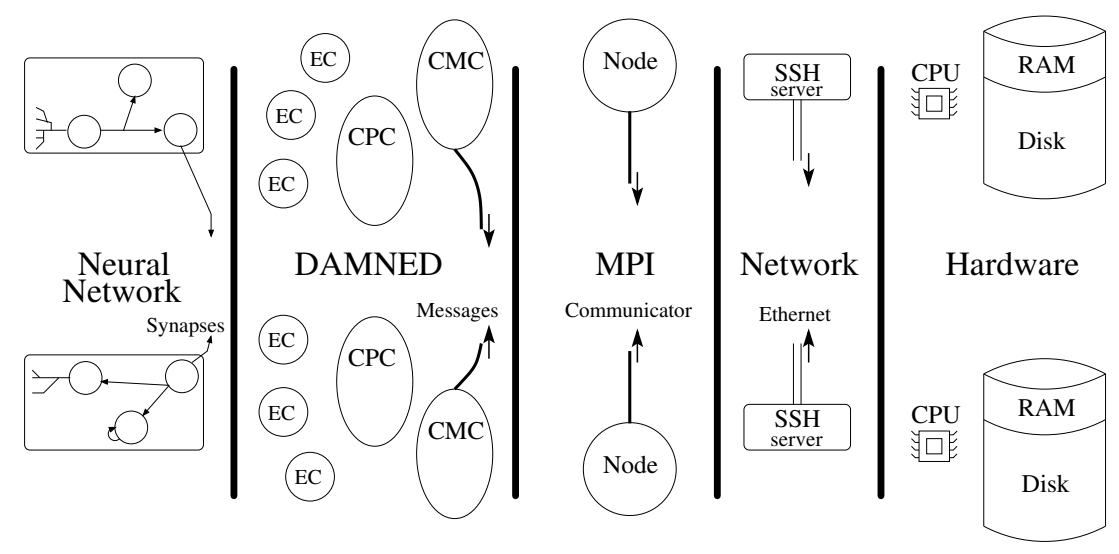

Fig. 1. Layered view for the global architecture of the DAMNED simulator.

\subsection{Mapping an SNN on DAMNED}

Through a user interface, the simulator allows the definition of network structures based on specification of populations and projections between them. User defined models of neuron, synapse and projection can be registered in the simulator through the interface. An environment process gives input stimulations to the simulated network and gets back output activities. This behaviour lets the environment free to use neural activities during the simulation, for instance to drive motor commands of robotic devices. At the end of the simulation the user may also access spikes, membrane potentials or synaptic weights and display their evolution during the simulation. DAMNED simulates the network activity in discrete time with an event-driven strategy [8]. The time scale used for simulation is up to the user. In present experiments the time scale is $0.1 \mathrm{~ms}$. Mapping the SNN onto the hardware is also user defined through the simulator interface. Each neuron is identified by an index and ranges of neuron indexes are associated with each simulation node.

\section{Model of Saccade Generation}

\subsection{Physiological Background}

In the brain, the neural network that generates motor commands for orienting the line of sight (i.e. to displace the eye to orient the fovea) toward stimulus of interest is among the best understood sensorimotor system, notably in its output part. The superior colliculus (SC), a paired and layered structure located at the roof of the brainstem (Fig. 2), plays a pivotal role to control saccadic eye displacement. Each SC controls saccades toward targets in the contralateral visual hemifield. The intermediate and deep layers of this structure are organized in a motor map with the amplitude and the direction of the movement respectively represented on its rostro-caudal and medio-lateral axes. Activities of several cortical areas involved in gaze orientation (Lateral Intra-Parietal area, 
Frontal Eye Field, Supplementary Eye Field) converge on this motor map. Collicular neurons send their axons toward premotor centers housing neurons called "Excitatory Burst Neurons" (EBNs). These neurons in turn directly control motoneurons of extraocular muscles.

Importantly, EBNs form an homogeneous pool without topography: The amplitude of the saccade is coded through the frequency and duration of the discharge. Hence, a spatial to temporal transformation has to be performed between the SC and the premotor centers. This spatio-temporal transformation is performed through a synaptic density gradient as anatomically observed between SC and EBNs $[9,10]$ : neurons located in the SC rostral pole make less synaptic boutons than those in the caudal pole (graded strength). In addition, the amplitude of the movement is controlled by a feedback loop [2] driving EBNs to discharge until a desired displacement signal is nullified.

The central mesencephalic reticular formation (cMRF) is another structure involved in gaze control. It is reciprocally connected with the SC [11] and projects notably toward a particular pool of neurons called omnipause neurons (OPNs) [4]. These OPNs are neurons that strongly inhibit EBNs. They are tonically active during fixation and silent during saccades. The shut off of their discharge is required for the initiation of the saccade. Given cMRF afferences from the SC and projections toward the OPNs, some neurons in this structure could be inhibitory interneurons that inhibit OPNs to trigger the saccade initiation.

To sum up, activity at the level of the SC is transmitted to at least two downstream structures: cMRF and EBNs. Finally, it is also known that the cMRF receives feedback from the EBNs.

\subsection{Theoretical Model}

Several papers proposed schemes including the cMRF but to our best knowledge no model including the cMRF has been yet implemented and tested. In previous schemes, roles assigned to the cMRF mostly concern the regulation and the update of SC activity. Indeed connections between SC and cMRF are reciprocal [11]. We propose that the cMRF could in addition be the point of comparison of the feedback loop.

In the present paper, for the sake of simplification, only the ouput part of the network is modelled. This simplification corresponds in fact to an experimental situation of electrical stimulation of the SC that evokes saccadic eye movements similar to natural ones $[12,13]$. We based our model on the following characteristics of the saccadic system. First, the feedback loop closes downstream of the SC [14]. Second, rather than organizing the feedback loop around a single output pathway from the SC, like for already proposed models [5], we suggest that one output pathway of the SC (the cMRF) could contain the desired displacement signal and that another one would be the forward branch of the organization through EBNs toward motoneurons. In addition, the EBNs activity would also be fed back to cMRF through an inhibitory interneuron. When the activity in cMRF neurons (the desired displacement) is reduced to zero through the feedback coming from EBNs, the inhibition of OPNs is removed and the saccade stops. 

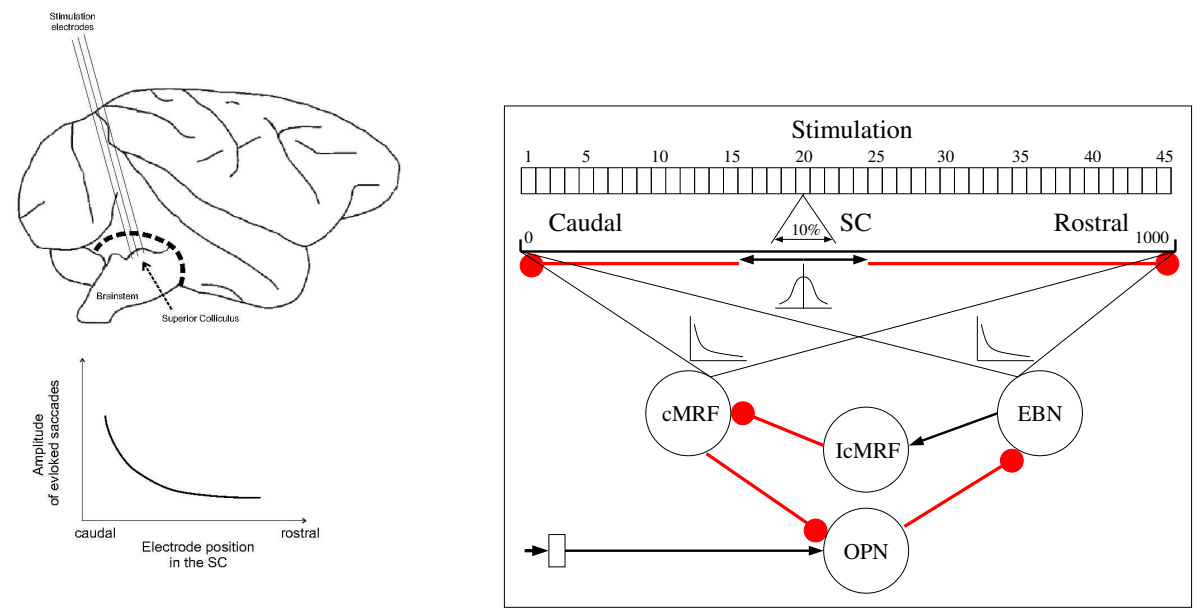

Fig. 2. Left : Anatomical locating of the Superior Colliculus at the top of the brainstem (top) and relationship between the amplitude of evoked saccades and the position of an electrical stimulation (bottom). Right : Topology of the modular spiking neural network. Black arrows show excitatory influence and red round ended arrows show inhibitory influence.

\subsection{Modular Spiking Neuron Network}

A modular spiking neuron network is proposed for implementing the theoretical model of saccade generation (Figure 2). The sub-collicular feedback loop relies on the interaction between three neuron populations. First, a population made of 100 EBNs which show a burst activity during saccade. Second a population of 100 OPNs which are tonically active before and after a saccade. Third, a population of $100 \mathrm{cMRF}$ neurons. OPNs and cMRF populations are inhibitory and EBNs population is excitatory. An inhibitory intermediate 100 neurons population (IcMRF) between EBNs and cMRF is introduced to implement the inhibitory influence of EBNs upon cMRF. OPNs inhibit EBNs and receive a constant external input activity in order to maintain a tonic activity before and after a saccade. cMRF in turn inhibits OPNs to trigger saccade initiation and ending.

As OPNs shut off under cMRF influence, the hyperpolarisation of EBN neurons is released. EBNs emit a burst of action potential indicating the saccade velocity (frequency of the burst). The discharge ends when the EBNs inhibition on cMRF, mediated by IcMRF, becomes stronger than the SC influence on cMRF. The saccade amplitude is determined by the frequency and duration of the EBNs burst.

As shown in Figure 2, a unidimensionnal 1000 neurons population of the Superior Colliculus (SC) is implemented and projects on both EBNs and cMRF populations. This SC population represents a rostro-caudal line in the right colliculus. The projection weights from SC to EBNs and $\mathrm{cMRF}$ are exponentially decreasing from the caudal to the rostral pole of the SC map. Consequently a range of horizontal saccade amplitudes to the left can be generated by such a saccade generation model. Excitatory gaussian shaped lateral excitation and long range inhibition are implemented in the collicular 
map to generate activation in a subpart of the SC map depending on the position of the stimulation.

Stimulations of the SC map at predefined positions are achieved by a 45 electrodes array. In the model each electrode is implemented as a cell connected to a subpart of the SC map. Stimulations are applied by making these cells fire spikes like impulsions to the connected neurons. The neuron model implemented in this network is a conductance based leaky integrate and fire neuron (LIF) [15].

\section{Experiments and Results}
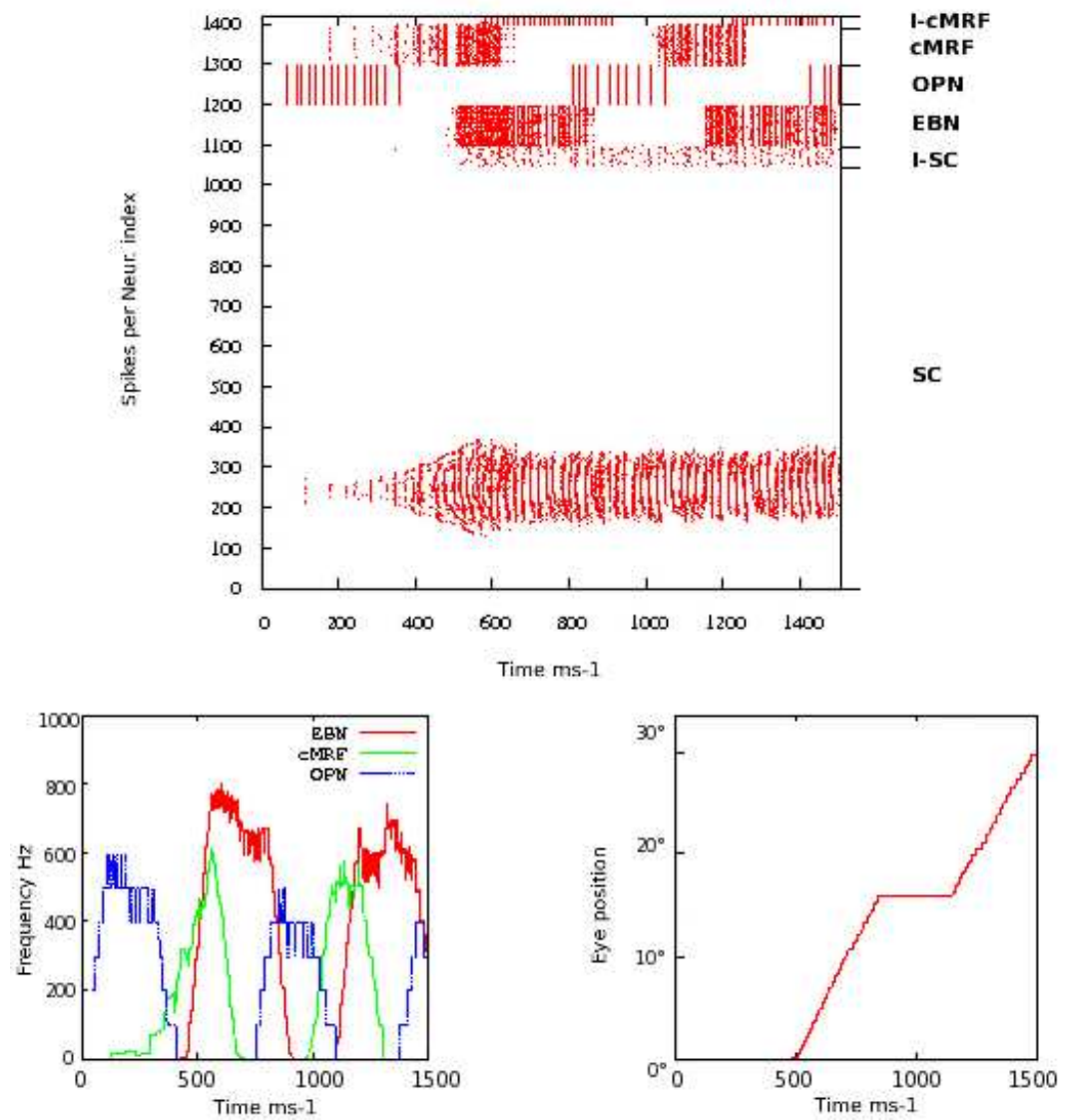

Fig. 3. Example of a generated saccade. Top : spike raster plot for each neuron function of time. Bottom left : instantaneous discharge frequencies of OPNs, EBNs and cMRF populations (averaged for all neurons in the populations). Bottom right : horizontal eye displacement in terms of number of EBNs spikes through time. 


\subsection{Experimental Protocols}

The electrical microstimulation of SC evokes saccadic gaze shifts similar to natural ones $[12,13]$. This artificial situation implies that the feedbacks to SC, for example from the cMRF, are neglected.

Each of the 45 electrodes generates an excitatory potential on the targeted neurons on the SC map. The impact range of the electrodes reaches $10 \%$ of the map around the electrode (Fig. 2). A competition between lateral excitations and long range inhibitions in the SC recruits about $20 \%$ of the map when the stimulation occurs. A supplementary electrode stimulates the OPNs during the whole experiment.

Stimulations on the SC are applied during $150 \mathrm{~ms}$ with a frequency set to $300 \mathrm{~Hz}$ and generates an activity in the map of 500 to $600 \mathrm{~Hz}$ on average. The OPNs population receives a constant external input at $400 \mathrm{~Hz}$ during the same period. An example of the network model response to stimulation is shown on Figure 3. Time is in $\mathrm{ms}^{-1}$.

\subsection{Stimulation Position}

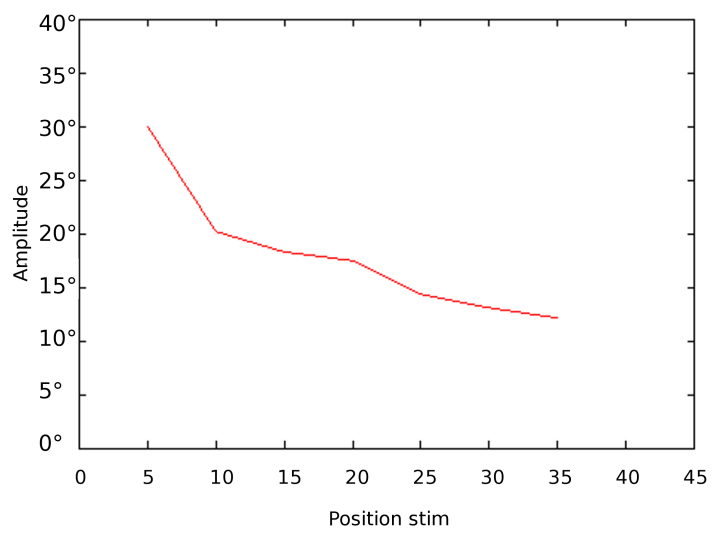

Fig. 4. Saccade amplitude function of the position on the SC map. Position is given in terms of the electrodes indexes.

The network model have been stimulated at seven different sites on the SC map to evaluate its ability to reproduce saccade amplitudes observed in biological microstimulation experiments. Saccade amplitude is computed from the number of fired EBNs spikes. The EBNs discharge frequency represents the eye velocity (Fig. 3). Despite the sustained stimulation on the SC map, saccades terminate with an amplitude that depends on the stimulation site. A caudal stimulation leads to a larger saccade amplitude than more rostral stimulations (Fig. 4). 
The non-linearity of the relationship between stimulation site position and saccade amplitude that is found in experimental studies is also present in our model. The SNN dynamic simulation confirms that the phenomenon is a logical consequence of the exponential weight gradient of the projection between the SC map and EBNs.

\subsection{Stimulation Parameters}

Both current intensity and pulse frequency variations have been observed in the model. The influence of these variations has been tested on two stimulation sites on the electrodes array : position 10 and position 30 . Current intensity in the model is given by the weight $(w)$ and radius ( $r$ in \% of the SC map) of the electrodes impact on the SC map. In standard conditions, the values of these parameters are set to $w=3.7$ and $r=5$ and allow to elicit a range of saccade amplitudes comparable to those obtained in biological studies (Figure 4). For the sake of comparison with experimental studies, let us label this condition as the $2 \times T$ condition, where $T$ is the threshold value of the couple $(w, r)$ necessary to elicit a saccade in response to electrical input. In order to study the behaviour of the model when varying current intensity, the model has been stimulated with three distinct intensities : $1,3 \times T(w=2.8, r=3.25), 2 \times T(w=3.7, r=5)$ and $6 \times T(w=11, r=15)$. The saccade amplitudes obtained are shown on Figure 5 (left).
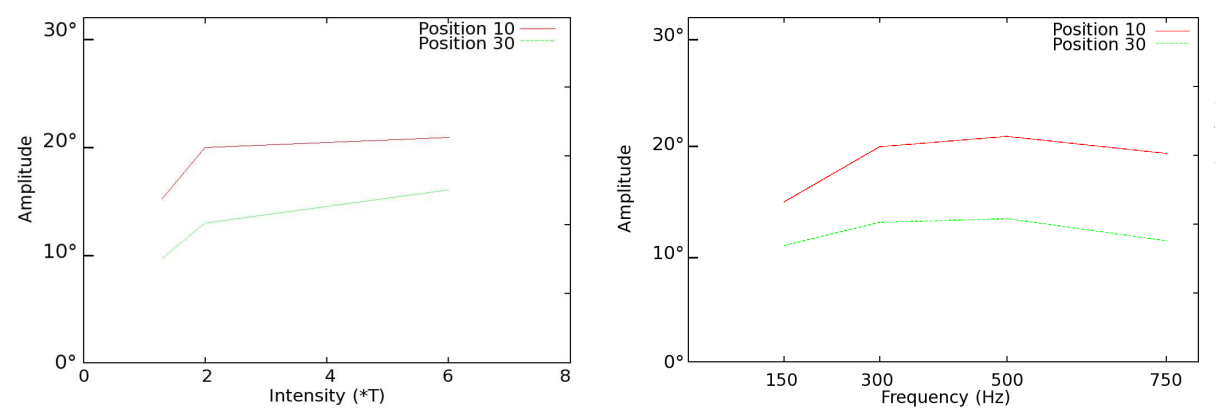

Fig. 5. Left : Saccade amplitude function of the stimulus intensity. Right : Saccade amplitude function of the stimulation frequency. Stimulations are applied at sites 10 and 30 on the electrode array.

Decreasing the current intensity $(1,3 \times T)$ leads to a decrease in saccade amplitude. Conversely, increasing the intensity $(6 \times T)$ does not result in a clear substantial increase in saccade amplitude. This pattern is in accordance with biological observations.

Tested stimulation pulse frequencies range from $150 \mathrm{~Hz}$ to $750 \mathrm{~Hz}$. The stimulation frequency in control condition was set to $300 \mathrm{~Hz}$. Results show that from $300 \mathrm{~Hz}$ to $500 \mathrm{~Hz}$, the amplitude of evoked saccades remains constant (Fig. 5, right). If the frequency is lowered (below $300 \mathrm{~Hz}$ ) or increased (beyond $500 \mathrm{~Hz}$ ), the saccade amplitude decreases. 


\section{Discussion}

A new organization of the neural network controlling saccadic eye movements has been proposed. The behaviour of the theoretical model has been simulated through time by implementing several neural populations in a modular spiking neuron network. The dynamics of the SNN has been observed and analyzed in event-driven simulations computed with the DAMNED simulator.

The control of saccadic eye movements is supposed to rely on a mechanism of feedback loop. Initially the required movement is coded on a motor map that is present in the SC. This collicular activity is transmitted to two downstream structures, the cMRF and the EBNs. The cMRF would control the movement initiation and contain a signal of desired displacement. The activity of the EBNs would produce the eye displacement and would be fed back to decrease the cMRF activity. When the activity in the cMRF is terminated, the eye movement stops. We show that our proposition based on two output pathways from the SC - one handling the desired displacement and one corresponding to a direct drive toward pre-motor neurons - is defensible when implemented with a modular SNN. Indeed, experimental results in simulation account for the classical results observed for electrical stimulations in the SC.

Despite its simplicity, the present scheme integrates the two major known projections from the SC to CMRF and to EBNs [11]. Given the implementation of projection gradients, moving the stimulation site toward the caudal pole of the SC leads to an increase in the amplitude of evoked saccades. The well-established organization in motor map is respected. Regarding the influence of the current intensity used for electrical stimulations, it is classically observed that increasing this parameter leads to a first phase of saccade amplitude increase, followed by a saturation when the site specific amplitude is reached (the amplitude for which cells under the electrode discharge for natural saccades) [12]. This pattern of amplitude evolution as a function of current intensity is obtained in the present work. The amplitude saturation is due to a saturation in the development of the activity at the level of the SC, itself due to intrinsic connectivity in the map (short range excitation and long range inhibition). Concerning frequency variation, results are also qualitatively similar to biological ones: A first phase of amplitude increase is followed by a saturation and later by an amplitude decrease [13]. This observation could result from local interactions in the SC, delays and/or refractory period of the LIF neuron model implemented. Interestingly, in the saturation range, the increase in stimulation frequency results in an increase of the displacement velocity. This is also an observation made in biological experiments.

The scheme proposed in the present paper remains very simple and several improvements could be considered. For example, the well described projection from the cMRF to the SC have been neglected since simulations have been limited to the condition of electrical stimulations. In future work, these projections would be added to capture the dynamics of the network in the situation of natural saccades toward visual target.

In conclusion, the DAMNED simulator has proved to be an excellent tool for testing by SNN implementation the dynamic behaviour of a new scheme for the organization of the neural network controlling saccade generation. Simulations have shown that our theoretical model accurately accounts for the dynamics of neural activities involved in the control of saccadic eye movements. 


\section{Acknowledgments}

This work has been partially supported by the ANR “MAPS”, grant BLAN07-1_185594.

\section{References}

1. Mouraud, A., Paugam-Moisy, H., Puzenat, D.: A distributed and multithreaded neural event driven simulation framework. In: PDCN. (2006) 212-217

2. Robinson, D.A.: Oculomotor control signals. In G., L., y Rita P., B., eds.: Basic Mechanisms of Ocular Motility and their Clinical Implications. Pergamon, Oxford, UK (1975) 337-374

3. Büttner, U., Büttner-Ennever, J.A.: Present concepts of oculomotor organization. In B\&, J., ed.: Neuroanatomy of the Oculomotor System. Volume 151 of Prog. Brain Res. Elsevier (2006) 1-42

4. Horn, A.K.: The reticular formation. Prog. Brain Res. 151 (2006) 127-155

5. Girard, B., Berthoz, A.: From brainstem to cortex: Computational models of saccade generation circuitry. Prog. Neurobiol. 77(4) (November 2005) 215-251

6. Brette, R., Rudolph, M., Carnevale, N.T., Hines, M.L., Beeman, D., Bower, J.M., Diesmann, M., Morrison, A., Goodman, P., Friederick, H.J., Zirpe, M., Natschläger, T., Pecevski, D., Ermentrout, G.B., Djurfeldt, M., Lansner, A., Rochel, O., Vieville, T., Muller, E., Davidson, A., El Boustani, S., Destexhe, A.: Simulation of networks of spiking neurons: A review of tools and strategies. J. Comp. Neur. 23(3) (2007) 349-398

7. Mouraud, A., Puzenat, D.: Simulation of large spiking neural networks on distributed architectures, the "damned " simulator. In Palmer-Brown, D., Draganova, C., Pimenidis, E., Mouratidis, H., eds.: EANN. Volume 43 of Communications in Computer and Information Science. Springer Berlin Heidelberg (August 2009) 359-370

8. Watts, L.: Event-driven simulation of networks of spiking neurons. In Cowan, J.D., Tesauro, G., Alspector, J., eds.: Advances in Neural Information Processing System. Volume 6., MIT Press (1994) 927-934

9. Ottes, F., Van Gisbergen, J.A., Eggermont, J.: Visuomotor fields of the superior colliculus : a quantitative model. Vis. Res. 26(6) (1986) 857-873

10. Moschovakis, A.K., Kitama, T., Dalezios, Y., Petit, J., Brandi, A.M., Grantyn, A.A.: An anatomical substrate for the spatiotemporal transformation. J. Neurosci. 18(23) (1998) 10219-10229

11. Chen, B., May, P.J.: The feedback circuit connecting the superior colliculus and central mesencephalic reticular formation: a direct morphological demonstration. Exp. Brain Res. 131 (2000) 10-21

12. Robinson, D.A.: Eye movements evoked by collicular stimulation in the alert monkey. Vis. Res. 12 (1972) 1795-1808

13. Guillaume, A., Pélisson, D.: Gaze shifts evoked by electrical stimulation of the superior colliculus in the head-unrestrained cat. i. effect of the locus and of the parameters of stimulation. Eur. J. Neur. 14 (August 2001) 1331-1344

14. Kato, R., Grantyn, A., Dalezios, Y., Moschovakis, A.K.: The local loop of the saccadic system closes downstream of the superior colliculus. Neuroscience 143(1) (November 2006) 319-337

15. Brette, R.: Exact simulation of integrate-and-fire models with synaptic conductances. Neur. Comp. 18(8) (2006) 2004-2027 